\title{
Das Karzinom am ösophagogastralen Übergang
}

\author{
Arnulf H. Hölscher ${ }^{\mathrm{a}}$ Peter Malfertheiner ${ }^{\mathrm{b}}$ \\ ${ }^{a}$ Klinik und Poliklinik für Allgemein-, Viszeral- und Tumorchirurgie, Universitätsklinik Köln, \\ ${ }^{b}$ Klinik für Gastroenterologie, Hepatologie und Infektiologie, Otto-von-Guericke-Universität, Magdeburg, Deutschland
}

Der Übergangsbereich der Speiseröhre in den Magen ist eine anatomisch und funktionell sehr interessante Sphinkterregion des Verdauungstraktes, da auf kurzer Distanz starke Gegensätze bestehen. Die Innenschicht wechselt vom Plattenepithel zum Zylinderepithel und ein Hohlorgan mit geringer Sekretion geht über in ein Organ mit stärkster Sekretion, verbunden mit einem extremen Sprung des luminalen $\mathrm{pH}-$ Wertes. Genau an dieser Stelle grenzen die Brusthöhle mit negativem Druck und die Bauchhöhle mit positivem Druck aneinander. Der Übergangsbereich ist unruhig durch die dauernden Schluckepisoden und die Atemverschieblichkeit des direkt benachbarten Zwerchfells. Durch Nachlassen der Sphinkterkraft und Ausbildung von Hiatushernien können in diesem umschriebenen Areal Veränderungen auftreten, die starke funktionelle und anatomische Auswirkungen haben.

Genau in diesem kleinen Bereich mit so charakteristischen Gegensätzen entwickeln sich Karzinome, die ebenfalls von sehr unterschiedlicher Natur sind. Das sogenannte BarrettKarzinom stellt das Endstadium einer langwierigen gastroösophagealen Refluxkrankheit dar, während das subkardiale Magenkarzinom eher eine langfristige Konsequenz der Helicobacter-pylori-Infektion ist. Dazwischen liegen die eigentlichen Kardiakarzinome, die sogenannten Adenokarzinome des ösophagogastralen Übergangs (AEG) Typ II, die Merkmale beider vorgenannter Entitäten tragen. Auf dieser kurzen Distanz von nur $10 \mathrm{~cm}$ wechselt bei diesen Karzinomen auch die Betroffenheit der Geschlechter in der Relation Männer zu Frauen von 8:1 für das Ösophaguskarzinom zu 2:1 beim subkardialen Magenkarzinom.

Die Klassifikation in die drei AEG-Typen, die 1987 erstmals beschrieben wurde, ist vielfach diskutiert und kritisiert worden. Sie hat sich jedoch mangels Alternativen für die Diagnostik, die wissenschaftliche Auswertung und insbesondere als Grundlage für die Entscheidung über das chirurgische Resektionsausmaß sehr bewährt und wird inzwischen international angewandt.

Durch den guten endoskopischen Zugang zum ösophagogastralen Übergang können Mukosakarzinome mit modernen Techniken oft kurativ entfernt werden, da Lymphknotenmetastasen in diesem frühen Stadium nur sehr selten bestehen. Für fortgeschrittene Tumoren ist die radikale Chirurgie nach wie vor das Kernstück der Behandlung. Sie ist im Grenzgebiet zwischen Brusthöhle und Bauchhöhle schwierig und ergibt die besten Ergebnisse in entsprechenden Zentren mit hoher Fallzahl. Zur weiteren Verbesserung der Langzeitergebnisse werden bei T3- und T4-Tumoren mehrere Modalitäten zusammengeführt, insbesondere in Form der neoadjuvanten Chemotherapie oder Radiochemotherapie vor der Operation.

Die Empfehlungen zur Diagnostik und Behandlung der ösophagogastralen Karzinome sind in der aktuellen S3-Leitlinie erarbeitet worden und werden am Ende des vorliegenden Schwerpunktheftes dargestellt. Die weiteren Beiträge enthalten aktuelle Aspekte zur Genese der Karzinome, zu begleitenden molekularbiologischen Veränderungen, zur Epidemiologie und zur palliativen Therapie.

Die Karzinome des ösophagogastralen Übergangs nehmen zu. Die Topographie der Magenkarzinome verschiebt sich deutlich vom distalen zum proximalen Drittel und das Barrett-Karzinom stellt zurzeit das Malignom mit der höchsten Zuwachsrate aller Tumoren dar. Daher werden alle Disziplinen, die sich mit der Diagnostik und Therapie dieser interessanten Entität befassen, zunehmend damit konfrontiert werden. Um dem Leser dafür alle aktuellen Informationen zu vermitteln, ist dieses Schwerpunktheft zusammengestellt worden. Es soll dazu dienen, unsere gemeinsamen Patienten bestmöglich zu behandeln.

\section{KARGER \\ Fax +497614520714 \\ Information@Karger.de}

www.karger.com
(C) 2012 S. Karger GmbH, Freiburg $1662-6664 / 12 / 0282-0084 \$ 38.00 / 0$

Accessible online at:

www.karger.com/vim
Prof. Dr. med. Peter Malfertheiner

Klinik für Gastroenterologie, Hepatologie und Infektiologie Otto-von-Guericke-Universität

Leipziger Straße 44, 39120 Magdeburg, Deutschland

Tel. +49 391-6713100, Fax -6713105

peter.malfertheiner@med.ovgu.de 